\title{
Effect of "add-on" interventions on exercise training in individuals with COPD: a systematic review
}

\author{
Carlos A. Camillo ${ }^{1,2,8}$, Christian R. Osadnik ${ }^{1,3,4,5,8}$, Hans van Remoortel ${ }^{1,6}$, \\ Chris Burtin ${ }^{1,7}$, Wim Janssens ${ }^{2}$ and Thierry Troosters ${ }^{1,2}$
}

Affiliations: ${ }^{1} \mathrm{KU}$ Leuven, Dept of Rehabilitation Sciences, Leuven, Belgium. ${ }^{2}$ University Hospital Leuven, Respiratory Division and Rehabilitation, Leuven, Belgium. ${ }^{3}$ Monash University, Dept of Physiotherapy, Victoria, Australia. ${ }^{4}$ Institute for Breathing and Sleep, Victoria, Australia. ${ }^{5}$ Monash Health, Monash Lung and Sleep, Victoria, Australia. ${ }^{6}$ Belgian Red Cross, Flanders, Mechelen, Belgium. ${ }^{7}$ Hasselt University, Rehabilitation Research Centre, Biomedical Research Institute, Faculty of Medicine and Life Sciences, Diepenbeek, Belgium. ${ }^{8}$ Both authors contributed equally.

Correspondence: Thierry Troosters, Respiratory Rehabilitation and Respiratory Division, UZ Gasthuisberg, Herestraat 49 Bus 706, Onderwijs en Navorsing I, Labo Pneumologie, B-3000 Leuven, Belgium.

E-mail: thierry.troostersamed.kuleuven.be

ABSTRACT The aim of this review was to identify the effectiveness of therapies added on to conventional exercise training to maximise exercise capacity in patients with chronic obstructive pulmonary disease (COPD).

Electronic databases were searched, identifying trials comparing exercise training with exercise training plus "add-on" therapy. Outcomes included peak oxygen uptake $\left(V^{\prime} \mathrm{O}_{2}\right.$ peak $)$, work rate and incremental/ endurance cycle and field walking tests. Individual trial effects on exercise capacity were extracted and collated into eight subgroups and pooled for meta-analysis. Sensitivity analyses were conducted to explore the stability of effect estimates across studies employing patient-centred designs and those deemed to be of "high" quality (PEDro score $>5$ out of 10 ).

74 studies (2506 subjects) met review inclusion criteria. Interventions spanned a broad scope of clinical practice and were most commonly evaluated via the 6-min walking distance and $V^{\prime} \mathrm{O}_{2}$ peak. Meta-analysis revealed few clinically relevant and statistically significant benefits of "add-on" therapies on exercise performance compared with exercise training. Benefits favouring "add-on" therapies were observed across six different interventions (additional exercise training, noninvasive ventilation, bronchodilator therapy, growth hormone, vitamin $\mathrm{D}$ and nutritional supplementation). The sensitivity analyses included considerably fewer studies, but revealed minimal differences to the primary analysis.

The lack of systematic benefits of "add-on" interventions is a probable reflection of methodological limitations, such as "one size fits all" eligibility criteria, that are inherent in many of the included studies of "add-on" therapies. Future clarification regarding the exact value of such therapies may only arise from adequately powered, multicentre clinical trials of tailored interventions for carefully selected COPD patient subgroups defined according to distinct clinical phenotypes.

@ERSpublications

Add-on modalities show mostly minimal additional benefits to exercise capacity after conventional training in COPD http://ow.ly/XABi4

Received: Oct 282015 | Accepted after revision: Jan 162016

Support statement: C.A. Camillo is a PhD fellow of the Ministério da Ciência, Tecnologia e Inovação, Conselho Nacional de Desenvolvimento Científico e Tecnológico (CNPq; Brazil), grant number 202425/2011-8. C.R. Osadnik is a recipient of a European Respiratory Society fellowship, grant number LTRF 2014-3132. T. Troosters is supported by the Flemish Research Foundation (Fonds Wetenschappelijk Onderzoek), grant number FWO G.0871.13. Funding information for this article has been deposited with FundRef.

Conflict of interest: None declared.

Copyright $\odot$ ERS 2016. This article is open access and distributed under the terms of the Creative Commons Attribution Non-Commercial Licence 4.0. 


\section{Introduction}

Pulmonary rehabilitation is a highly effective treatment for individuals with chronic obstructive pulmonary disease (COPD), and is associated with significant improvement in quality of life, healthcare utilisation and exercise capacity [1-3]. Benefits of exercise training include improvements in oxygen uptake and endurance time [4], reduced symptoms [1] and muscle composition adaptations such as increased capillary/fibre ratio [5] and increased oxidative capacity [6]. In recent years, significant attention has been directed towards improving the already beneficial effects of pulmonary rehabilitation via application of additional ("add-on") therapies on top of conventional exercise training. This is probably due to the emergence of newer therapies (such as pharmacological medicines), new exercise modalities and more readily available equipment (such as portable noninvasive ventilation (NIV) units). The effectiveness of such supplemental therapies, however, is unclear.

The only existing systematic review of such therapies was conducted over a decade ago [7]. Due to significant methodological limitations and small study sample sizes, the authors concluded there was insufficient evidence to estimate the value of most supplemental interventions except supplemental oxygen (which demonstrated a clear lack of benefit). The increased number of studies conducted since this review, however, offers greater potential to better estimate the usefulness of "add-on" therapy to exercise training. It is also crucial that the effects of interventions seeking to boost training responses are rigorously determined according to a robust range of end-points specific to the physiological adaptations of exercise in this patient group. This is likely to improve our understanding of the mechanisms explaining their role in exercise training, and potentially enhance their translation into clinical practice. If no further clarification is able to be determined regarding the value of "add-on" therapies to exercise training in patients with COPD, one must question the scientific approach to clinical research in this area and consider how best to address any emergent shortcomings in order to improve future studies and, ultimately, clinical patient care.

The principal aim of this review was to determine whether "add-on" therapies, applied in conjunction with conventional exercise training in pulmonary rehabilitation, improve exercise capacity in individuals with COPD more than conventional exercise training alone. The secondary aim was to examine the effectiveness of "add-on" therapies specifically in studies that adopted highly specific, patient-centred approaches to the study design. As pulmonary rehabilitation benefits are driven largely by physiological adaptation of skeletal muscles in response to exercise training, we focused on a comprehensive range of outcomes specifically related to exercise capacity.

\section{Methods}

\section{Study selection criteria}

Randomised and quasi-randomised controlled clinical trials were eligible for inclusion if they compared exercise training (comprising aerobic (lower limb endurance) training) with exercise training plus at least one additional treatment modality (no exclusions) in patients with COPD (defined by study authors). Studies were considered quasi randomised if participants were assigned to interventions by methods such as sequential allocation, date of birth, etc. In line with the clear physiological mechanisms underpinning exercise training effects in COPD, studies were only included in the review if they reported data from at least one of the following exercise capacity outcomes: peak oxygen uptake ( $V^{\prime} \mathrm{O}_{2}$ peak; metabolic equivalents), peak work rate (watts), cycle endurance test (time), incremental shuttle walk test (ISWT; distance, time), endurance shuttle walk test (ESWT; time, distance), 6-min walk test (6MWT; distance) and 12-min walk test (12MWT; distance). Outcomes such as quality of life or symptoms were not considered for this review.

\section{Search strategy}

Six databases (CENTRAL, MEDLINE, EMBASE, SciELO, PEDro and CINAHL) were searched in April 2014 without limits using the following subject headings (no free text terms): (COPD OR chronic obstructive pulmonary disease) AND (randomised OR randomized) AND (exercis ${ }^{\star}$ OR exercise training OR aerobic exercise training). Differences between database subject heading structures (e.g. MeSH versus Emtree) resulted in differences between some search terms across platforms. No hand searching of conference proceedings was undertaken. Search yields were exported to Reference Manager v12 (Thomson Reuters, Philadelphia, PA, USA) and duplicates discarded. Studies were scrutinised on title and abstract by two independent reviewers (C.A. Camillo and H. van Remoortel) and coded as either "include", "exclude" or "awaiting full text". Disagreements were resolved by an independent, third reviewer (C.R. Osadnik). Eligibility criteria were applied by two study investigators and further hand searching performed by examination of reference lists of included studies and comparison with the earlier systematic review [7]. Studies that included more than one relevant "add-on" therapy type (e.g. pulmonary rehabilitation versus pulmonary rehabilitation plus oxygen versus pulmonary rehabilitation plus nutrition) were included but represented once in the PRISMA (Preferred Reporting Items for Systematic Reviews and Meta-Analyses) flow diagram. 


\section{Data extraction and quality appraisal}

Data were extracted into an electronic spreadsheet using a standardised template. Study quality was assessed using the PEDro scale. This validated scale [8] rates internal and external validity according to 11 criteria, appraised according to standardised decision rules, to derive a total quality score out of 10 (higher=better; first item not quantified). No studies were excluded on the basis of quality. Where data were missing or unclear, attempts were made to contact authors via email. Study characteristics and quality were summarised and presented in tables.

\section{Analysis}

Data were pooled for meta-analysis in Review Manager v5.3.5 (The Nordic Cochrane Centre, The Cochrane Collaboration, Copenhagen, Denmark) according to eight clinically homogenous intervention groups. These were: 1) additional exercise training modalities, with subgroups for lower limb strength training, upper and lower limb strength training and other; 2) NIV; 3) oxygen; 4) heliox; 5) prescription medications, with subgroups for tiotropium, anabolic steroids, growth hormone, vitamin D and hypertonic saline; 6) nutrition, with subcategories for proteins/fats, creatine and amino acids; 7) breathing exercises, with subgroups of inspiratory muscle training and breathing retraining; and 8) other. A complete list of interventions included within each group classification is provided in table 1 . Data were entered as change from baseline values wherever possible. If change data were unable to be obtained (from the article or author contact), end-point (post-intervention) data were included in the meta-analysis in accordance with Cochrane guidelines [83].

Data were combined on an outcome-by-outcome basis using weighted mean differences of native metrics (e.g. metres, L. $\mathrm{min}^{-1}$, etc.) with a fixed effect model wherever possible. Standardised mean differences were used to pool data for outcomes containing multiple measurement types (e.g. walk test time and distance). A random effects model was used where statistical heterogeneity was "greater than moderate", indicated by an $\mathrm{I}^{2}$ statistic $>60 \%$ and Chi-squared $\mathrm{p}<0.05$. For studies that involved multiple "add-on" interventions, participant numbers in the control group were evenly divided for analysis according to the number of interventions, in order to derive accurate effect estimates and avoid data duplicity [83]. Data were not weighted or pooled across different intervention groups due to clinical heterogeneity regarding their application. Data were summarised in a meta-analysis matrix, with each cell representing the result of the pooled analysis result for a given intervention (row) on a given outcome (column). Data from individual studies may therefore appear more than once across columns. A representative forest plot was generated for the most commonly reported outcome (6MWT). The secondary aim of the study was addressed via meta-analysis of data originating only from studies that used highly specific eligibility criteria deemed well suited to the "add-on" intervention, in accordance with a patient-centred care model.

\section{Sensitivity analyses}

One sensitivity analysis was defined a priori and performed to determine the impact of studies identified as quasi randomised on effect estimates. A post hoc analysis was also undertaken to explore the impact of removing studies deemed to be low quality (PEDro score $\leqslant 5$ ) from the principal analysis. For both analyses, relevant studies were removed from the principal meta-analysis and findings compared between the original and subsequent analyses.

\section{Results}

From 2524 records identified in the database search, 74 studies of 2506 individuals with COPD met eligibility criteria and were included in the review (figure 1). Characteristics of the included studies are presented in table 1 and figure 2.

Three studies [24, 33, 81] reported data from more than one appropriate intervention arm. The methodological quality of the included studies was moderate (median (interquartile range) PEDro score 5 (4-7); table 2), due mainly to lack of intention-to-treat analysis $(n=63)$, therapist blinding $(n=55)$ and allocation concealment $(n=54)$. Significant statistical heterogeneity was only observed in five weighted analyses, namely: nutritional supplementation $\left(V^{\prime} \mathrm{O}_{2}\right.$ peak $\left.\mathrm{I}^{2}=63 \%\right)$, anabolic steroids supplementation $\left(V^{\prime} \mathrm{O}_{2}\right.$ peak $\left.\mathrm{I}^{2}=66 \%\right)$, NIV (cycled endurance time $\left.\mathrm{I}^{2}=74 \%\right)$, breathing retraining $\left(12 \mathrm{MWT} \mathrm{I}^{2}=74 \%\right)$ and growth hormone $\left(6 \mathrm{MWT} \mathrm{I}^{2}=82 \%\right)$. Inspection of study methodology and participant characteristics of these trials revealed minor differences that were not considered indications for exclusion from analysis. Meta-analysis of these data therefore proceeded with application of a random effects model (table 3). The duration of interventions varied largely, ranging from 10 to 50 sessions (mean \pm SD $27 \pm 19$ sessions) and exceptionally reaching 108 sessions (breathing exercises group). Studies typically reported data from one $(\mathrm{n}=46)$ or two $(\mathrm{n}=21)$ exercise outcomes, most commonly the 6MWT (figure 3) and $V^{\prime} \mathrm{O}_{2}$ peak. The mean rate of attrition from included studies was modest (table 1) but similar across intervention and control groups (11.4\% versus $9.6 \%$, respectively). 
TABLE 1 Description of studies included in the final screening

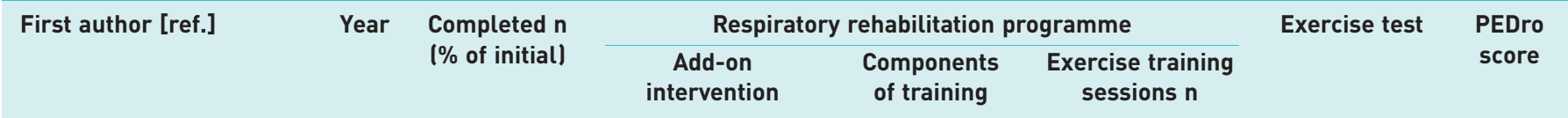

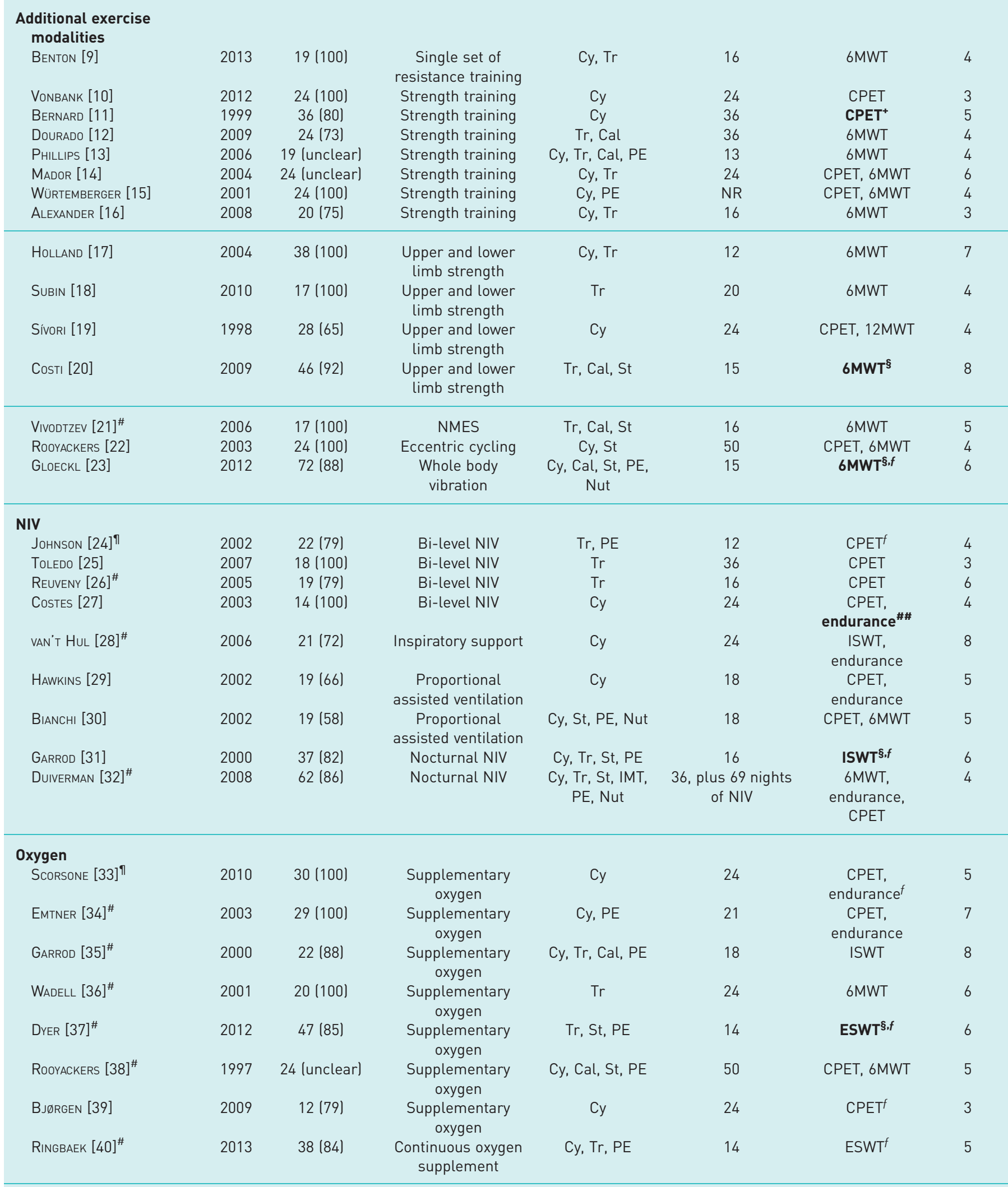


TABLE 1 Continued

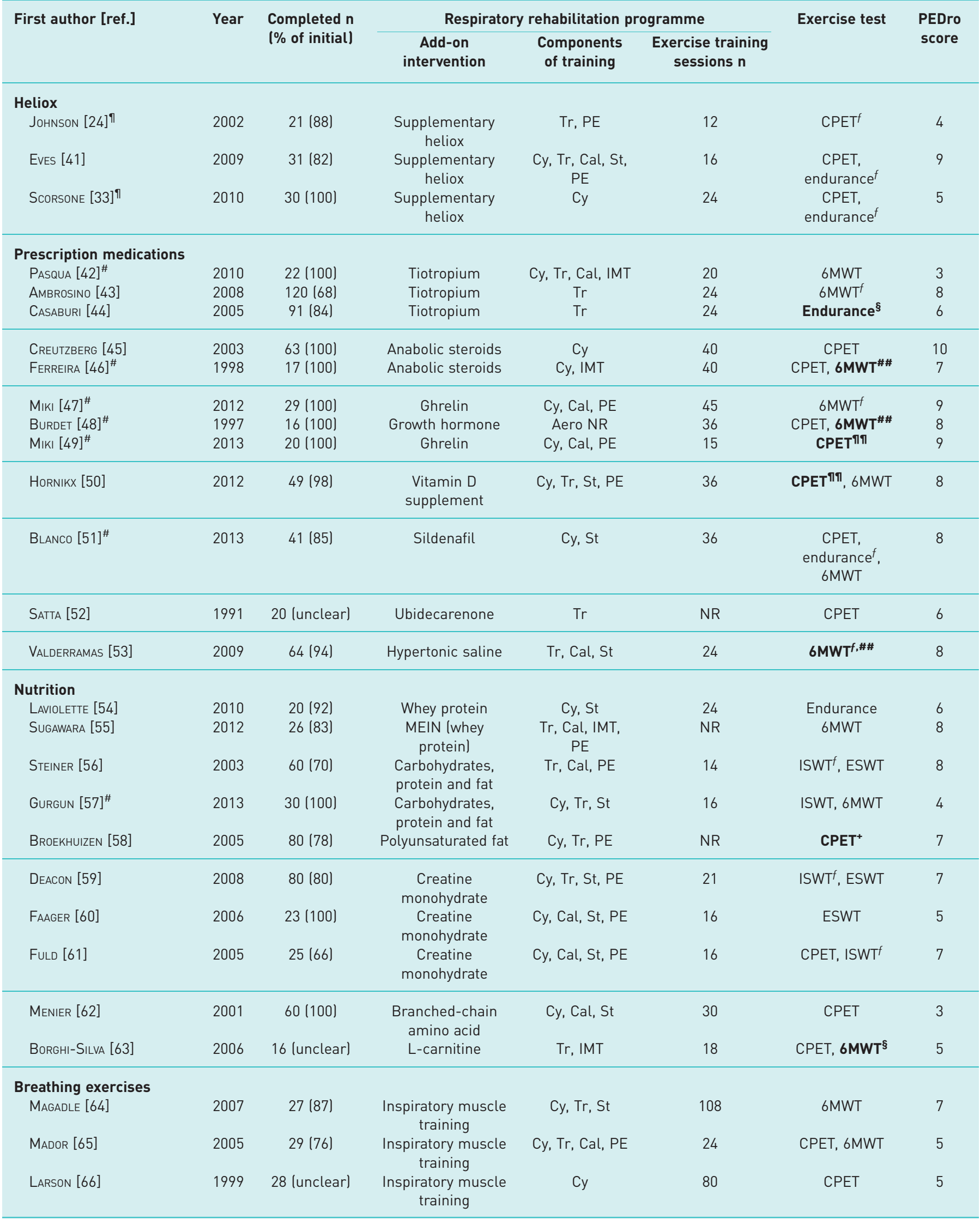




\section{TABLE 1 Continued}

\begin{tabular}{|c|c|c|c|c|c|c|c|}
\hline \multirow[t]{2}{*}{ First author [ref.] } & \multirow[t]{2}{*}{ Year } & \multirow{2}{*}{$\begin{array}{l}\text { Completed } \mathbf{n} \\
\text { [\% of initial] }\end{array}$} & \multicolumn{3}{|c|}{ Respiratory rehabilitation programme } & \multirow[t]{2}{*}{ Exercise test } & \multirow{2}{*}{$\begin{array}{l}\text { PEDro } \\
\text { score }\end{array}$} \\
\hline & & & $\begin{array}{c}\text { Add-on } \\
\text { intervention }\end{array}$ & $\begin{array}{l}\text { Components } \\
\text { of training }\end{array}$ & $\begin{array}{l}\text { Exercise training } \\
\text { sessions } \mathrm{n}\end{array}$ & & \\
\hline WANKE [68] & 1994 & 42 (unclear) & $\begin{array}{l}\text { Inspiratory muscle } \\
\text { training }\end{array}$ & Cy & $\begin{array}{l}\text { 32, plus } 56 \text { IMT } \\
\text { sessions }\end{array}$ & CPET & 4 \\
\hline DeKhuiJzen [70] ${ }^{\#}$ & 1991 & $40(100)$ & $\begin{array}{l}\text { Inspiratory muscle } \\
\text { training }\end{array}$ & Cy, Tr, St & 100 & CPET, 12MWT & 5 \\
\hline KUNIKOSHITA [71] & 2006 & $15(100)$ & $\begin{array}{l}\text { Inspiratory muscle } \\
\text { training }\end{array}$ & Tr, Cal & 18 & CPET & 4 \\
\hline SYKES [72] & 2005 & 37 (93) & $\begin{array}{l}\text { Inspiratory } \\
\text { muscle training }\end{array}$ & Cy, St & 40 & CPET, 6MWT & 7 \\
\hline Coluins [74] & 2008 & 33 (79) & $\begin{array}{l}\text { Ventilation } \\
\text { feedback }\end{array}$ & $\mathrm{Cy}, \mathrm{Tr}$ & 36 & $\begin{array}{l}\text { CPET, } \\
\text { endurance }\end{array}$ & 5 \\
\hline van GeStel [75] & 2012 & $40(100)$ & $\begin{array}{l}\text { Controlled } \\
\text { breathing }\end{array}$ & Cy, St & 10 & 6MWT & 6 \\
\hline \multicolumn{8}{|l|}{ Other } \\
\hline CARRIERI-Kohlman [76] & 1996 & $51(100)$ & $\begin{array}{l}\text { Coaching versus } \\
\text { monitoring }\end{array}$ & $\operatorname{Tr}$ & 12 & CPET, 6MWT & 5 \\
\hline ZANOTTI [77] & 2012 & $20(100)$ & Osteopathy & Cy, St, PE & 20 & $6 \mathrm{MWT}$ & 9 \\
\hline AleXANDER [78] & 2012 & 27 (unclear) & Harmonica playing & Cy, $\mathrm{Tr}$ & 16 & $6 \mathrm{MWT}$ & 4 \\
\hline DE Godoy [79] & 2003 & $30(100)$ & Psychotherapy & Cy, Tr, Cal, St & 24 & 6MWT & 5 \\
\hline
\end{tabular}

Results from the meta-analysis, represented as mean differences weighted according to outcome, are presented in table 3 and figure 3 (6MWT only).

\section{Primary aim}

Overall, the meta-analysis revealed few clinically relevant or statistically significant benefits of "add-on" therapy on exercise capacity compared with pulmonary rehabilitation. Significant benefits favouring "add-on" therapy were observed across seven different interventions ("other" additional exercise training, NIV, tiotropium, growth hormone, vitamin D, proteins/fats and amino acids) and five different outcomes (6MWT, $V^{\prime} \mathrm{O}_{2}$ peak, peak work rate, cycle endurance time and ISWT) (table 3, in bold). Most interventions were evaluated across more than one clinical outcome; however, no single intervention category demonstrated statistically significant benefits over multiple outcome measures (i.e. within rows, only one significant result in table 3 ).

Two statistically significant negative effects were observed on exercise capacity, measured by the 6MWT. Both were interventions within the "prescription medications" category. The mean between-group 


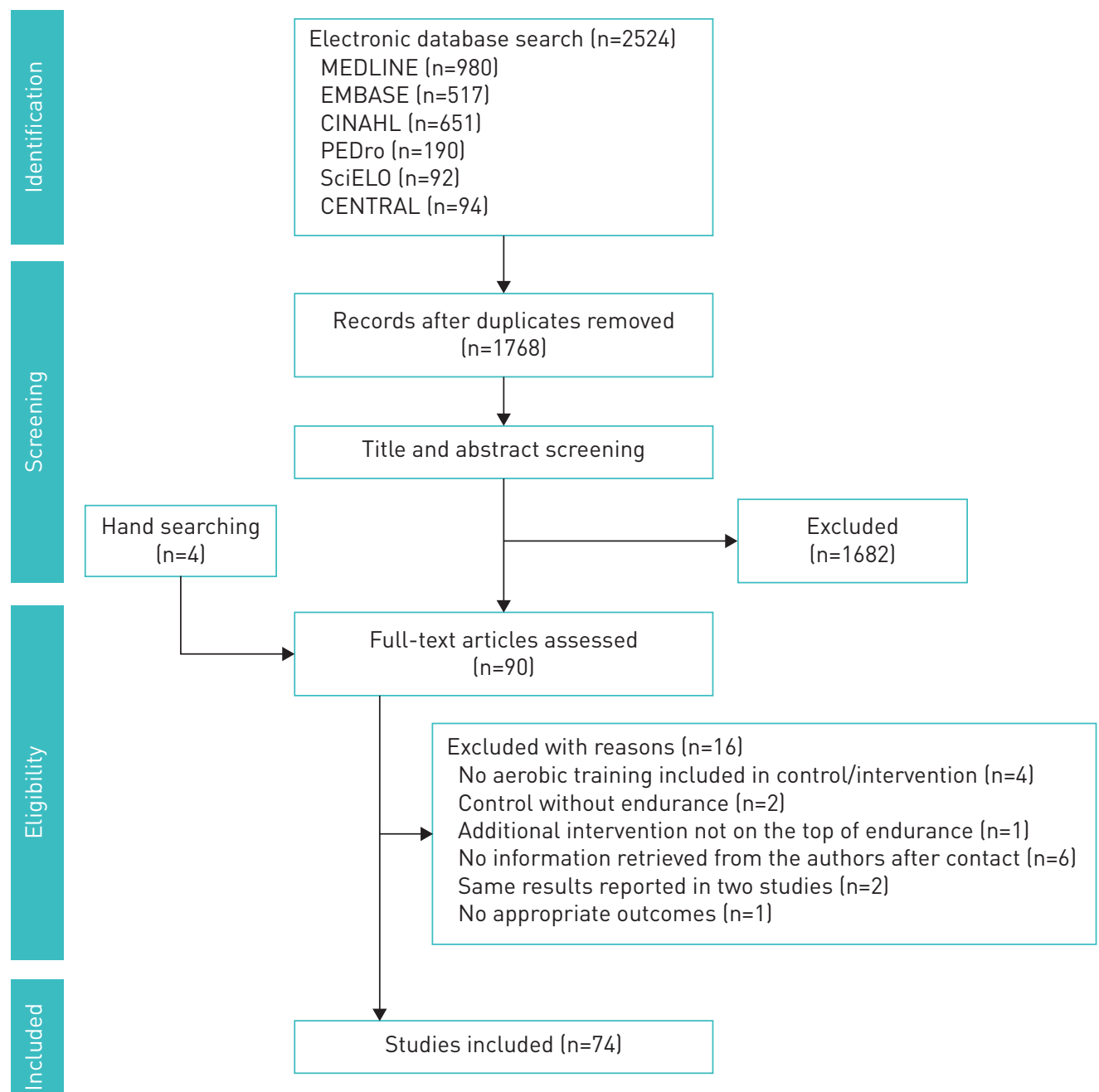

FIGURE 1 PRISMA (Preferred Reporting Items for Systematic Reviews and Meta-Analyses) flow diagram.

difference in 6MWT was $-190.0 \mathrm{~m}(95 \% \mathrm{CI}-247.34--132.66 \mathrm{~m} ; \mathrm{n}=57)$ for hypertonic saline, and $-63.00 \mathrm{~m}(95 \% \mathrm{CI}-91.09--34.91 \mathrm{~m} ; \mathrm{n}=17)$ for anabolic steroids.

A post hoc pooled meta-analysis of the three "additional exercise training" subcategories revealed an overall statistically significant improvement in 6MWT response (mean difference $18.41 \mathrm{~m}$; 95\% CI 9.18-27.63 m; 12 studies of 344 participants).

\section{Secondary aim}

18 studies used highly specific eligibility criteria, in accordance with a patient-centred care model (table 1). When considering these data in isolation, loss of statistical significance occurred for many outcomes (table 4). The two remaining significant findings were a positive effect of growth hormone on $V^{\prime} \mathrm{O}_{2}$ peak and a negative

FIGURE 2 Number of studies, according to year and region, which compared exercise training with exercise training plus "add-on" in the present review.

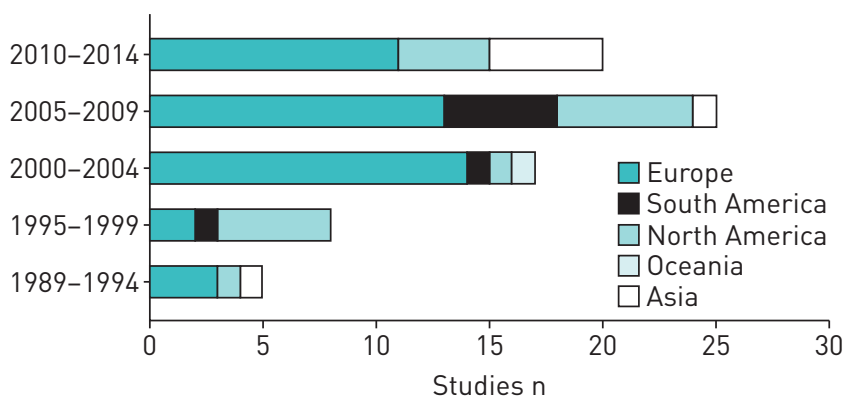


TABLE 2 Overview of methodological quality (PEDro score) of included studies

First author [ref.]

PEDro criteria

\begin{tabular}{llllllllllll}
\hline $1 \#$ & 2 & 3 & 4 & 5 & 6 & 7 & 8 & 9 & 10 & 11 & Total
\end{tabular}

\section{Additional exercise modalities}

Benton [9]

VonBANK [10]

Bernard [11]

DouRADo [12]

PHILLIPS [13]

MADOR [14]

WÜRTEMBERGER [15]

AleXANDER [16]

HOLLAND [17]

SUBIN [18]

SívoRI [19]

Cost। [20]

Vivodtzev [21]"

ROOYACKERS [22]

GLOECKL [23]

NIV

JoHNSON $[24]^{\S}$

ToLedo [25]

REUVENY [26]"

COSTES [27]

VAN 'T HUL [28]"

HaWkINS [29]

BIANCHI [30]

GARROD [31]

Duiverman [32]"

\section{Oxygen}

SCORSONE [33] $]^{\S}$

EMTNER [34]"

GARROD [35]

WADELL [36] ]"

DYER [37]

RooYACKERS [38]"

BJøRGEN [39]

RingBaEk [40]"

Heliox

JoHnson [24] $]^{\S}$

EVES [41]

Scorsone [33] $]^{\S}$

\section{Prescription medications}

Pasqua [42]"

Ambrosino [43]

CASABURI [44]

Creutzberg [45]

FerReIRA [46]"

MІKI [47]"

BURDET [48]

MIKI [49]"

HORNIKX [50]

BLANCO [51]"

SATTA [52]

VALDERRAMAS [53]

\section{Nutrition}

LAVIOLETTE [54]

SugaWARA [55]

StEINER [56]

GuRgun [57]

BROEKHUIZEN [58]

DeAcon [59]
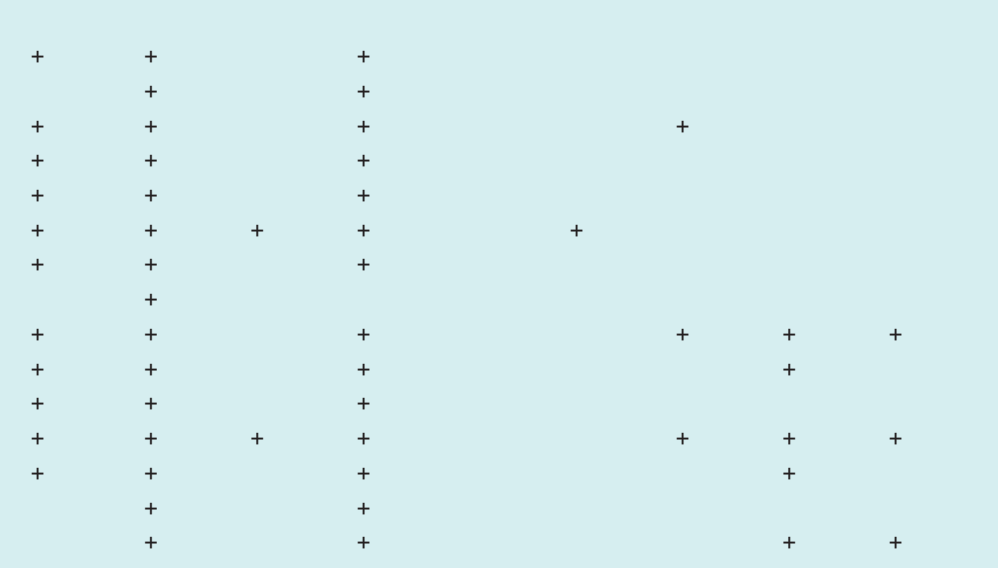

$\begin{array}{lll}+ & + & 4 \\ & + & 3 \\ + & + & 5 \\ + & + & 4 \\ + & + & 4 \\ + & + & 6 \\ + & + & 4 \\ + & + & 3 \\ + & + & 7 \\ & + & 4 \\ + & + & 4 \\ + & + & 8 \\ + & + & 5 \\ + & + & 4 \\ + & + & 6\end{array}$


TABLE 2 Continued

\begin{tabular}{|c|c|c|c|c|c|c|c|c|c|c|c|c|}
\hline \multirow[t]{2}{*}{ First author [ref.] } & \multicolumn{12}{|c|}{ PEDro criteria } \\
\hline & $1^{\#}$ & 2 & 3 & 4 & 5 & 6 & 7 & 8 & 9 & 10 & 11 & Total \\
\hline FAAGER [60] & + & + & & & + & & & + & & + & + & 5 \\
\hline Menier [62] & & & & + & & & & + & & & + & 3 \\
\hline BoRGHI-SILVA [63] & & + & & + & + & & & & & + & + & 5 \\
\hline \multicolumn{13}{|l|}{ Breathing exercises } \\
\hline LARSON [66] & + & + & & + & & & + & & & + & + & 5 \\
\hline BERRY [67] & + & + & & & & & + & + & & + & + & 5 \\
\hline WANKE [68] & & + & + & & & & & & & + & + & 4 \\
\hline WEINER [69] & & + & & + & & & & + & & + & + & 5 \\
\hline DeKhUIJZEN [70]" & + & + & & + & & & & + & & + & + & 5 \\
\hline KUNIKOSHITA [71] & & + & & + & & & & & & + & + & 4 \\
\hline \multicolumn{13}{|l|}{ Other } \\
\hline CARRIERI-KoHLMAN [76] & + & + & & + & & & & + & & + & + & 5 \\
\hline ZANOTTI [77] & + & + & + & + & + & & + & + & + & + & + & 9 \\
\hline ALEXANDER [78] & + & + & + & & & & & & & + & + & 4 \\
\hline DE Godor [79] & + & + & & + & & & & + & & + & + & 5 \\
\hline SHARIFABAD [80] & + & + & & + & & & & & & + & + & 4 \\
\hline NoRWEG $[81]^{\S}$ & + & + & & & & & & & & + & + & 3 \\
\hline DeERING [82] & + & + & & + & & & + & & + & + & + & 6 \\
\hline
\end{tabular}

1: eligibility criteria specified; 2: randomisation; 3: concealed allocation; 4: groups similar at baseline; 5 : blinding of subjects; 6: blinding of therapists; 7: blinding of assessors; 8: at least one key outcome with $>85 \%$ of initially allocated subjects; 9: intention-to-treat analysis; 10 : between-group statistical comparison; 11: study provides both point measures and measures of variability for at least one key outcome; NIV: noninvasive ventilation; +: criteria decision rule satisfied. ${ }^{\#}$ : item does not contribute to overall score; ${ }^{\text {ๆ: }}$ exemplar studies of patient-centred care; ${ }^{\S}$ : study included two appropriate interventions.

effect of anabolic steroids on 6MWT. Most findings from this analysis related to data from single and reportedly underpowered studies.

\section{Sensitivity analysis}

The removal of quasi-randomised trials $(n=2)[27,62]$ did not affect any treatment effect estimates. Findings from the principal analysis were also largely unchanged when studies deemed to be of "low" overall quality (PEDro score $\leqslant 5$ ) were removed from the analysis. The only two changes of statistical significance were the loss of benefit of nutrition supplementation (amino acids) on 6MWT due to a lack of available data, and emergence of a large, statistically significant benefit of oxygen therapy on ESWT from one individual study (mean difference $490.00 \mathrm{~m}$; 95\% CI $237.90-742.10 \mathrm{~m}$; one study of 47 participants). These data are presented in table 5 .

\section{Discussion}

This review comprehensively demonstrates that, on average, "add-on" modalities rarely enhance exercise capacity responses to conventional exercise training when applied to a general COPD population. This "evidence of lack of effect" is different from the previous "lack of evidence of effect" observed by PUHAN et al. [7]. Our findings demonstrate consistency with the recent British Thoracic Society guideline on pulmonary rehabilitation, which yielded minimal supportive evidence-based recommendations regarding the value of select adjunct therapies in pulmonary rehabilitation [84], but expand the scope of findings across a broader range of interventions including different pharmacological interventions and additional exercise training modalities. The latter is particularly important given the high global variation in approaches to exercise training for patients with COPD. The lack of observed benefits in this review does not imply that "add-on" therapies have no role for the management of patients with COPD undertaking exercise training. Statistical significance was rarely observed in our large data synthesis; however, data from some intervention categories (table 3 ) may provide insight into potentially useful strategies to guide future 
TABLE 3 Summary of treatment effect estimates, weighted according to outcome

\begin{tabular}{|c|c|c|c|c|c|c|c|}
\hline & $\begin{array}{l}V^{\prime} 0_{2 \text { peak }} \\
L \cdot \mathrm{min}^{-1}\end{array}$ & $\begin{array}{l}\text { Peak work rate } \\
\text { W }\end{array}$ & $\begin{array}{l}\text { Cycle endurance } \\
\text { min }\end{array}$ & $\begin{array}{l}\text { ISWT } \\
\text { m }\end{array}$ & ESWT & $\begin{array}{l}\text { 6MWT } \\
\mathrm{m}\end{array}$ & $\begin{array}{l}\text { 12MWT } \\
\mathrm{m}\end{array}$ \\
\hline \multicolumn{8}{|l|}{ Additional exercise training } \\
\hline LL strength & $\begin{array}{l}0.10(-0.08-0.29) ; \\
\mathrm{N}^{2}=32 i \text { versus } 28 \mathrm{c}\end{array}$ & $\begin{array}{l}3.64(-4.95-12.30) \\
\mathrm{N}^{4}=54 i \text { versus } 54 \mathrm{c}\end{array}$ & & & & $\begin{array}{l}2.50(-20.63-25.64) ; \\
\mathrm{N}^{6}=62 i \text { versus } 68 \mathrm{c}\end{array}$ & \\
\hline UL/LL strength & $\begin{array}{l}0.00(-0.18-0.16) ; \\
N^{1}=14 i \text { versus } 14 c\end{array}$ & & & & & $\begin{array}{c}23.36(-4.59-51.32)^{\#} ; \\
N^{3}=54 i \text { versus } 47 \mathrm{c}\end{array}$ & $\begin{array}{l}4.78(-122.43-131.99) ; \\
N^{1}=14 i \text { versus } 14 \mathrm{c}\end{array}$ \\
\hline Other & $\begin{array}{l}0.10(-0.31-0.51) \\
N^{1}=12 i \text { versus } 12 c\end{array}$ & $\begin{array}{l}-2.00(-44.24-42.24) \\
N^{1}=12 i \text { versus } 12 \mathrm{c}\end{array}$ & & & & $\begin{array}{l}24.43(0.13-48.74) ; \\
N^{3}=57 i \text { versus } 56 \mathrm{c}\end{array}$ & \\
\hline NIV & $\begin{array}{l}0.08(-0.08-0.24) ; \\
\mathrm{N}^{3}=25 i \text { versus } 26 \mathrm{c}\end{array}$ & $\begin{array}{l}-0.03(-7.55-7.50) ; \\
\mathrm{N}^{4}=35 i \text { versus } 36 \mathrm{c}\end{array}$ & $\begin{array}{l}0.61(-5.23-6.46)^{\#} ; \\
\mathrm{N}^{3}=27 i \text { versus } 25 \mathrm{c}\end{array}$ & $\begin{array}{l}23.71(2.65-44.77) ; \\
\mathrm{N}^{2}=27 \mathrm{i} \text { versus } 31 \mathrm{c}\end{array}$ & & $\begin{array}{c}-17.35(-48.39-13.70) ; \\
N^{2}=39 i \text { versus } 42 c\end{array}$ & \\
\hline Oxygen & $\begin{array}{l}-0.11(-0.29-0.07) ; \\
\mathrm{N}^{3}=36 i \text { versus } 32 \mathrm{c}\end{array}$ & $\begin{array}{c}-1.38(-5.47-2.72) ; \\
\mathrm{N}^{3}=36 i \text { versus } 32 \mathrm{c}\end{array}$ & $\begin{array}{l}4.70(-1.96-11.36) ; \\
N^{1}=14 i \text { versus } 15 \mathrm{c}\end{array}$ & & $\begin{array}{c}233.38(-245.24-712.0) \mathrm{m}^{\#} ; \\
\mathrm{N}^{2}=40 \mathrm{i} \text { versus } 45 \mathrm{c}\end{array}$ & $\begin{array}{c}-37.0(-98.61-24.61) ; \\
N^{1}=12 i \text { versus } 12 \mathrm{c}\end{array}$ & \\
\hline Heliox & $\begin{array}{l}0.02(-0.04-0.08) ; \\
\mathrm{N}^{2}=26 i \text { versus } 20 \mathrm{c}\end{array}$ & $\begin{array}{l}4.61(-0.50-9.72) ; \\
\mathrm{N}^{2}=26 i \text { versus } 20 \mathrm{c}\end{array}$ & $\begin{array}{l}5.20(-0.17-10.57) \\
\mathrm{N}^{1}=16 i \text { versus } 15 \mathrm{c}\end{array}$ & & & & \\
\hline \multicolumn{8}{|l|}{ Prescription medications } \\
\hline Tiotropium & & & $\begin{array}{c}5.35(0.89-9.81) ; \\
\mathrm{N}^{1}=47 \mathrm{i} \text { versus } 44 \mathrm{c}\end{array}$ & & & $\begin{array}{l}7.06(-15.13-29.26) ; \\
N^{2}=68 i \text { versus } 74 c\end{array}$ & \\
\hline Anabolic steroids & $\begin{array}{l}0.10(-0.02-0.23) \\
N^{1}=33 i \text { versus } 30 c\end{array}$ & $\begin{array}{l}4.00(-5.28-13.28) ; \\
N^{1}=33 i \text { versus } 30 \mathrm{c}\end{array}$ & & & & $\begin{array}{c}-63.0(-91.09--34.91) ; \\
N^{1}=10 i \text { versus } 7 \mathrm{c}\end{array}$ & \\
\hline Growth hormone & $\begin{array}{c}0.05(0.01-0.09) ; \\
\mathrm{N}^{2}=18 \mathrm{i} \text { versus } 18 \mathrm{c}\end{array}$ & $\begin{array}{c}-11.00(-26.48-4.48) ; \\
N^{1}=8 i \text { versus } 8 \mathrm{c}\end{array}$ & & & & $\begin{array}{l}-26.21(-148.92-96.50)^{\#} ; \\
N^{2}=22 i \text { versus } 23 c\end{array}$ & \\
\hline Vitamin D & $\begin{array}{c}0.64(0.07-1.22) ; \\
\mathrm{N}^{1}=25 \mathrm{i} \text { versus } 24 \mathrm{c}\end{array}$ & $\begin{array}{l}7.00(-0.28-14.28) ; \\
N^{1}=25 i \text { versus } 24 c\end{array}$ & & & & $\begin{array}{l}29.0(-7.62-65.62) ; \\
\mathrm{N}^{1}=25 i \text { versus } 24 \mathrm{c}\end{array}$ & \\
\hline Hypertonic saline & & & & & & $\begin{array}{c}-190.0 \\
(-247.34--132.66) ; \\
N^{1}=30 i \text { versus } 27 \mathrm{c}\end{array}$ & \\
\hline \multicolumn{8}{|l|}{ Nutritional supplementation } \\
\hline Proteins/fats & $\begin{array}{l}-0.03(-0.12-0.06) ; \\
\mathrm{N}^{1}=38 i \text { versus } 42 \mathrm{c}\end{array}$ & $\begin{array}{l}9.00(4.32-13.68) ; \\
\mathrm{N}^{1}=38 \mathrm{i} \text { versus } 42 \mathrm{c}\end{array}$ & $\begin{array}{l}2.86(-2.00-7.72) \\
N^{1}=10 i \text { versus } 10 \mathrm{c}\end{array}$ & $\begin{array}{c}-19.48(-42.23-3.27) \\
N^{2}=40 i \text { versus } 50 \mathrm{c}\end{array}$ & $\begin{array}{c}-0.24(-1.65-1.17) \mathrm{min} ; \\
N^{1}=15 i \text { versus } 15 \mathrm{c}\end{array}$ & $\begin{array}{l}8.02(-31.21-47.26) ; \\
N^{2}=31 \text { versus } 25 \mathrm{c}\end{array}$ & \\
\hline Creatine & $\begin{array}{l}0.14(-0.02-0.29) ; \\
N^{1}=14 i \text { versus } 11 \mathrm{c}\end{array}$ & $\begin{array}{l}4.29(-9.53-18.11) ; \\
\mathrm{N}^{1}=14 i \text { versus } 11 \mathrm{c}\end{array}$ & & $\begin{array}{c}-3.62(-31.75-24.52) ; \\
N^{2}=52 i \text { versus } 53 \mathrm{c}\end{array}$ & $\begin{array}{c}-0.11(-0.46-0.24) \text { units }^{\pi} ; \\
\mathrm{N}^{3}=65 i \text { versus } 63 \mathrm{c}\end{array}$ & & \\
\hline Amino acids & & $\begin{array}{c}-0.90(-14.55-12.75) \\
N^{1}=30 i \text { versus } 30 \mathrm{c}\end{array}$ & & & & $\begin{array}{c}53.00(24.09-81.91) ; \\
N^{1}=8 i \text { versus } 8 \mathrm{c}\end{array}$ & \\
\hline $\begin{array}{l}\text { Breathing exercises } \\
\text { Inspiratory muscle training }\end{array}$ & $\begin{array}{c}0.03 \\
(-0.26-0.31) \text { units } \\
\mathrm{N}^{6}=97 \mathrm{i} \text { versus } 95 \mathrm{c}\end{array}$ & $\begin{array}{c}-0.07(-10.56-10.43) \\
\mathrm{N}^{4}=70 i \text { versus } 69 \mathrm{c}\end{array}$ & & & & $\begin{array}{c}12.72(-16.21-42.26) ; \\
N^{4}=55 i \text { versus } 49 \mathrm{c}\end{array}$ & $\begin{array}{c}211.45 \\
(-56.66-479.56)^{\#} ; \\
\mathrm{N}^{3}=39 i \text { versus } 41 \mathrm{C}\end{array}$ \\
\hline Breathing retraining & $\begin{array}{l}0.20(-1.58-1.98) ; \\
N^{1}=17 i \text { versus } 16 \mathrm{c}\end{array}$ & & $\begin{array}{l}8.50(-4.38-21.38) ; \\
N^{1}=17 i \text { versus } 16 \mathrm{c}\end{array}$ & & & $\begin{array}{c}-12.58 \text { (-35.93-10.77); } \\
N^{1}=20 i \text { versus } 20 \mathrm{c}\end{array}$ & \\
\hline Other & $\begin{array}{l}-0.28(-0.83-0.27) ; \\
\mathrm{N}^{1}=24 i \text { versus } 27 \mathrm{c}\end{array}$ & & & $\begin{array}{l}3.20(-24.06-30.46) ; \\
N^{1}=16 i \text { versus } 25 c\end{array}$ & & $\begin{array}{c}10.26(-21.85-42.38) \\
\mathrm{N}^{6}=78 \mathrm{i} \text { versus } 183 \mathrm{c}\end{array}$ & \\
\hline
\end{tabular}




\begin{tabular}{|c|c|c|c|c|c|c|c|c|}
\hline \multirow[t]{2}{*}{ Subgroups and studies } & \multirow[t]{2}{*}{ Intervention type } & \multicolumn{2}{|c|}{ Participants n } & \multirow{2}{*}{$\begin{array}{c}\text { Other reported } \\
\text { outcomes }\end{array}$} & \multirow{2}{*}{$\begin{array}{c}\text { Weight } \\
\%\end{array}$} & \multirow{2}{*}{$\begin{array}{c}\text { Mean difference } \\
{[95 \% \mathrm{CI}]}\end{array}$} & & \\
\hline & & Intervention & Control & & & & & \\
\hline \multicolumn{9}{|c|}{ Additional exercise training } \\
\hline \multicolumn{9}{|c|}{ Lower limb strength training } \\
\hline DouRADo [12] & Strength training & 11 & 13 & & 21.1 & $17.00(-33.35-67.35)$ & & - \\
\hline PHILLIPS [13] & Strength training & 10 & 9 & & 16.3 & $6.10(-51.22-63.42)$ & & - \\
\hline BENTON [9] & Single set of resistance training & 10 & 9 & & 17.2 & $3.50(-52.27-59.27)$ & & - \\
\hline MADOR [14] & Strength training & 11 & 13 & CPET & 5.6 & $-4.60(-102.05-92.85)$ & & \\
\hline WÜRTEMBERGER [15] & Strength training & 10 & 14 & CPET & 33.8 & $-5.40(-45.21-34.41)$ & & \\
\hline ALEXANDER [16] & Strength training & 10 & 10 & & 6.0 & $-10.05[-104.79-84.69]$ & & \\
\hline Subtotal $(95 \% \mathrm{CI})$ & & 62 & 68 & & 100.0 & $2.50(-20.63-25.64)$ & & \\
\hline \multicolumn{9}{|c|}{ Upper and lower limb training } \\
\hline Costi [20] & Upper and lower limb strength & 23 & 23 & & 34.3 & $50.20(28.87-71.53)$ & & -- \\
\hline SUBIN [18] & Upper and lower limb strength & 9 & 8 & & 38.6 & $10.40(-3.68-24.48)$ & & \\
\hline HoLLAND [17] & Upper and lower limb strength & 22 & 16 & & 27.1 & $7.90(-24.61-40.41)$ & & \\
\hline Subtotal $(95 \% \mathrm{CI})$ & & 54 & 47 & & 100.0 & $23.36(-4.59-51.32)^{\#}$ & & \\
\hline \multicolumn{9}{|l|}{ Other training } \\
\hline VIVODTZEV [21] & Whole body vibration & 9 & 8 & & 6.7 & $39.00(-54.57-132.57)$ & & \\
\hline GLOECKL [23] & NMES & 36 & 36 & & 89.1 & $26.70(0.94-52.46)$ & & - \\
\hline ROOYACKERS [22] & Eccentric cycling & 12 & 12 & CPET & 4.2 & $-47.00(-165.60-71.60)$ & & \\
\hline Subtotal $(95 \% \mathrm{CI})$ & & 57 & 56 & & 100.0 & $24.43(0.13-48.74)$ & & \\
\hline \multicolumn{9}{|l|}{ NIV } \\
\hline Duiverman [32] & Nocturnal NIV & 30 & 32 & Endurance, CPET & 30.0 & $15.00(-41.69-71.69)$ & & - \\
\hline BIANCHI [30] & Proportional assist ventilation & 9 & 10 & CPET & 70.0 & $-31.20(-68.30-5.90)$ & & \\
\hline Subtotal $(95 \% \mathrm{CI})$ & & 39 & 42 & & 100.0 & $-17.35(-48.39-13.70)$ & & \\
\hline \multicolumn{9}{|l|}{ Oxygen supplementation } \\
\hline ROOYACKERS [38] & Supplemental oxygen & 12 & 12 & CPET & NA & $-37.00(-98.61-24.61)$ & & - \\
\hline \multicolumn{9}{|l|}{ Prescription medications } \\
\hline AMBROSINo [43] & Tiotropium & 57 & 63 & & 91.1 & $7.30(-15.96-30.56)$ & & \\
\hline PASQUA [42] & Tiotropium & 11 & 11 & & 8.9 & $4.66(-69.63-78.95)$ & & \\
\hline Subtotal $(95 \% \mathrm{CI})$ & & 68 & 74 & & 100.0 & $7.06(-15.13-29.26)$ & & \\
\hline FERREIRA [46] & Anabolic steroids & 10 & 7 & CPET & NA & $-63.00(-91.09--34.91)$ & & \\
\hline MIKI [47] & Growth hormone & 14 & 15 & & 56.3 & $29.00(-10.69-68.69)$ & & \\
\hline BURDET [48] & Growth hormone & 8 & 8 & CPET & 43.7 & $-97.21(-193.28--1.14)$ & & \\
\hline Subtotal $(95 \% \mathrm{Cl})$ & & 22 & 23 & & 100.0 & $-26.21(-148.92-96.50)^{\#}$ & & \\
\hline HoRNIKX [50] & Vitamin D supplementation & 25 & 24 & CPETी & NA & $29.00(-7.62-65.62)$ & & + \\
\hline VALDERRAMAS [53] & Hypertonic saline & 30 & 27 & & NA & $-190.00(-247.34--132.66)$ & + & \\
\hline \multicolumn{9}{|c|}{ Nutritional supplementation } \\
\hline SUGAWARA [55] & MEIN (whey protein) & 16 & 10 & & 3.7 & $82.70(-38.59-203.99)$ & & \\
\hline BoRGHI-SILVA [63] & L-carnitine & 8 & 8 & CPET & 64.8 & $53.00(24.09-81.91)$ & & - \\
\hline GURGUN [57] & Carbohydrates, protein and fat & 15 & 15 & ISWT & 31.5 & $-0.70(-42.16-40.76)$ & & - \\
\hline Subtotal $(95 \% \mathrm{CI})$ & & 39 & 33 & & 100.0 & $37.17(13.89-60.44)$ & & $>$ \\
\hline \multicolumn{9}{|l|}{ Breathing exercises } \\
\hline Inspiratory muscle trair & & & & & & & & \\
\hline SYKES $[72]$ & Inspiratory muscle training & 20 & 17 & CPET & 38.3 & $35.40(-12.33-83.13)$ & & $-1-$ \\
\hline MagadLe [64] & Inspiratory muscle training & 14 & 13 & & 18.6 & $17.90(-50.58-86.38)$ & & \\
\hline GoLDSTEIN [73] & Inspiratory muscle training & 6 & 5 & Endurance & 28.1 & $-4.00(-59.74-51.74)$ & & - \\
\hline MADOR [65] & Inspiratory muscle training & 15 & 14 & CPET & 15.0 & $-20.20(-96.36-55.96)$ & & \\
\hline Subtotal $(95 \% \mathrm{CI})$ & & 55 & 49 & & 100.0 & $12.72(-16.81-42.26)$ & & \\
\hline Breathing retraining & & & & & & & & \\
\hline VAN GeStel [75] & Controlled breathing & 20 & 20 & & NA & $-12.58(-35.93-10.77)$ & & \\
\hline Other & & & & & & & & \\
\hline ZANOTTI [77] & Osteopathy & 10 & 10 & & 17.1 & $64.80(-12.94-142.54)$ & & 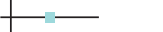 \\
\hline ALEXANDER [78] & Harmonica playing & 9 & 18 & & 4.6 & $39.24(-110.32-188.80)$ & & 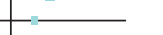 \\
\hline DE Godor [79] & Psychotherapy & 14 & 16 & & 24.5 & $35.30(-29.63-100.23)$ & & -1 \\
\hline NoRWEg [81] & Activity training & 11 & 6 & & 14.5 & $19.63(-64.80-104.06)$ & & - \\
\hline NoRWEg [81] & Lectures & 10 & 6 & & 14.2 & $-17.52(-102.70-67.66)$ & & - \\
\hline CARRIERI-KOHLMAN [76] & Coaching versus monitoring & 24 & 27 & CPET & 25.2 & $-46.02(-110.02-17.98)$ & & \\
\hline Subtotal $(95 \% \mathrm{Cl})$ & & 78 & 83 & & 100.0 & $10.26(-21.85-42.38)$ & & \\
\hline & & & & & & & $\begin{array}{c}100-100 \\
\text { Favours } \\
\text { control }\end{array}$ & $\begin{array}{c}0 \quad 100200 \\
\text { Favours } \\
\text { experimental }\end{array}$ \\
\hline
\end{tabular}

FIGURE 3 Forest plot of the effect of "add-on" therapies on 6-min walking distance change (in metres). Effect estimates (mean differences) without coloured shading denote unweighted analyses of data from single studies within intervention groups or subgroups. CPET: cardiopulmonary cycle exercise test; NMES: neuromuscular electrical stimulation; NIV: noninvasive ventilation; NA: not available; ISWT: incremental shuttle walk test. \#: random effects model used for meta-analysis; " : statistically significant positive effect reported.

research. For example, the magnitude and direction of effect estimates (although nonsignificant) were consistent across all except one study within the "additional exercise training modalities" category for $V^{\prime} \mathrm{O}_{2}$ peak, peak work rate, $6 \mathrm{MWT}$ and $12 \mathrm{MWT}$. The magnitude of estimated mean improvement in 6MWT following both "upper and lower limb strength" and "other" training subcategories was also close to the threshold of the minimally important difference (MID) for this outcome [85, 86]. Further research may therefore be warranted to investigate the potential for additional exercise training modalities to enhance exercise training effects, in more select patient subgroups. Our post hoc pooled analysis of the three 
TABLE 4 Summary of standardised effect estimates for studies defined as "exemplar" models of patient-centred care

$V^{\prime} 0_{2}$ peak

Peak work rate

Cycle endurance

ISWT

ESWT

6MWT

12MWT

$L \cdot \min ^{-1}$

W

$\min$

m

\section{Additional exercise training}

LL strength

UL/LL strength

Other

NIV

Oxygen

$0.15(-0.29-0.59)$ units"
$\mathrm{N}^{2}=39 i$ versus $42 \mathrm{c}$

\section{$8.00(-5.53-21.53):$}

$\mathrm{N}^{1}=9 \mathrm{i}$ versus $10 \mathrm{c}$

$3.50(-1.38-8.38)$

$17.00(-5.47-39.47) ;$
$N^{1}=10 i$ versus $11 c$

$39.00(-54.57-132.57)$

$\mathrm{N}^{1}=9 \mathrm{i}$ versus $8 \mathrm{c}$

$-4.52(-17.07-8.03) ; \quad 4.70(-1.96-11.36)$;

$\mathrm{N}^{2}=26 \mathrm{i}$ versus $27 \mathrm{c} \quad \mathrm{N}^{1}=14 \mathrm{i}$ versus $15 \mathrm{c}$

5.00 (-41.69-71.69);

$\mathrm{N}^{1}=30 \mathrm{i}$ versus $32 \mathrm{c}$

Heliox

Prescription medications

Tiotropium

Anabolic steroids

\section{$-0.59(-1.58-0.40)$ units";}

$\mathrm{N}^{1}=10 \mathrm{i}$ versus $7 \mathrm{c}$

$\mathrm{N}^{1}=11 \mathrm{i}$ versus $11 \mathrm{c}$

Growth hormone

$0.05(0.01-0.09)$;

$-63.0(-91.09--34.91) ;$

$\mathrm{N}^{1}=10 \mathrm{i}$ versus $7 \mathrm{c}$

Vitamin D

Hypertonic saline

Nutritional supplementation

Proteins/fats

Creatine

Amino acids

\section{Breathing exercises}

Inspiratory muscle training

$-0.06(-0.19-0.07) ; \quad-7.00(-32.90-18.90)$

$69.00(-154.89-292.89)$

$\mathrm{N}^{1}=20 \mathrm{i}$ versus $20 \mathrm{c} \quad \mathrm{N}^{1}=20 \mathrm{i}$ versus $20 \mathrm{c}$
$\mathrm{N}^{1}=20 \mathrm{i}$ versus $20 \mathrm{c}$

Breathing retraining

\section{Other}

Data are presented as mean difference (95\% CI), where the mean difference is the change from baseline and a positive effect estimate favours intervention ("add-on" therapies). Data from individual studies may appear more than once across different outcomes (columns). $V^{\prime} \mathrm{O}_{2}$ peak: peak oxygen uptake; ISWT: incremental shuttle walk test; ESWT: endurance shuttle walk test; 6MWT: 6-min walk test; 12MWT: 12-min walk test; LL: lower limb; UL: upper limb; NIV: noninvasive ventilation; N: number of participants (x is number of studies); walk test; $6 \mathrm{MWT}$ : 6-min walk test; ${ }^{1}$ intervention group; $\mathrm{c}$ : control group. ${ }^{\#}$ : random effects model; ${ }^{9}$ : standardised mean difference. Bold indicates statistically significant treatment effect.

$\begin{array}{ccc}-26.00(-72.28-20.28) ; & -0.12(-0.84-0.6) \text { units } & -0.70(-42.16-40.76) ; \\ N^{1}=15 i \text { versus } 15 c & N^{1}=15 i \text { versus } 15 c & N^{1}=15 i \text { versus } 15 c\end{array}$

$.21(-148.92-96.50)^{\#}$

$\mathrm{N}^{2}=22 \mathrm{i}$ versus $23 \mathrm{c}$ 
TABLE 5 Sensitivity analysis: summary of treatment effect estimates, weighted according to outcome, including studies with PEDro scores $>5$ only

\begin{tabular}{|c|c|c|c|c|c|c|c|}
\hline & $\begin{array}{l}V^{\prime} o_{2_{\text {peak }}} \\
\mathrm{L} \cdot \mathrm{min}^{-1}\end{array}$ & $\begin{array}{c}\text { Peak work rate } \\
\text { W }\end{array}$ & $\begin{array}{l}\text { Cycle endurance } \\
\text { min }\end{array}$ & $\begin{array}{l}\text { ISWT } \\
\mathrm{m}\end{array}$ & ESWT & $\begin{array}{c}\text { 6MWT } \\
\mathrm{m}\end{array}$ & $\begin{array}{c}12 \mathrm{MWT} \\
\mathrm{m}\end{array}$ \\
\hline \multicolumn{8}{|l|}{ Additional exercise training } \\
\hline LL strength & $\begin{array}{l}-0.10(-0.43-0.23) ; \\
\mathrm{N}^{1}=11 \text { versus } 13 \mathrm{c}\end{array}$ & $\begin{array}{l}9.00(-14.60-32.60) ; \\
N^{1}=11 i \text { versus } 13 c\end{array}$ & & & & $\begin{array}{c}-4.60(-102.05-92.85) ; \\
N^{1}=11 \text { versus } 13 \mathrm{c}\end{array}$ & \\
\hline UL/LL strength & & & & & & $\begin{array}{l}30.90(-10.39-72.20)^{\#} ; \\
N^{2}=45 i \text { versus } 39 \mathrm{c}\end{array}$ & \\
\hline Other & & & & & & $\begin{array}{l}26.70(0.94-52.46) ; \\
\mathrm{N}^{1}=36 \mathrm{i} \text { versus } 36 \mathrm{c}\end{array}$ & \\
\hline NIV & $\begin{array}{l}0.08(-0.15-0.32) \\
N^{1}=9 i \text { versus } 10 c\end{array}$ & $\begin{array}{l}8.00(-5.53-21.53) ; \\
N^{1}=9 i \text { versus } 10 c\end{array}$ & $\begin{array}{l}3.50(-1.38-8.38) \\
N^{1}=10 i \text { versus } 11 \mathrm{c}\end{array}$ & $\begin{array}{l}23.71 \text { (2.65-44.77); } \\
\mathrm{N}^{2}=27 \mathrm{i} \text { versus } 31 \mathrm{c}\end{array}$ & & & \\
\hline Oxygen & $\begin{array}{l}-0.04(-0.26-0.18) ; \\
\mathrm{N}^{0}=14 \mathrm{i} \text { versus } 15 \mathrm{c}\end{array}$ & $\begin{array}{c}3.00(-16.32-22.32) \\
N^{1}=14 i \text { versus } 15 \mathrm{c}\end{array}$ & $\begin{array}{l}4.70(-1.96-11.36) ; \\
N^{1}=14 i \text { versus } 15 c\end{array}$ & & $\begin{array}{c}490.00(237.90-742.10) \mathrm{m} ; \\
\mathrm{N}^{1}=24 \mathrm{i} \text { versus } 23 \mathrm{c}\end{array}$ & & \\
\hline Heliox & $\begin{array}{l}0.02(-0.04-0.08) ; \\
N^{2}=16 i \text { versus } 15 \mathrm{c}\end{array}$ & $\begin{array}{l}4.00(-4.15-12.15) ; \\
N^{1}=16 i \text { versus } 15 c\end{array}$ & $\begin{array}{l}5.20(-0.17-10.57) ; \\
N^{1}=16 i \text { versus } 15 c\end{array}$ & & & & \\
\hline \multicolumn{8}{|l|}{ Prescription medications } \\
\hline Tiotropium & & & $\begin{array}{c}5.35(0.89-9.81) ; \\
\mathrm{N}^{1}=47 \mathrm{i} \text { versus } 44 \mathrm{C}\end{array}$ & & & $\begin{array}{l}7.30(-15.96-30.56) ; \\
N^{1}=57 i \text { versus } 63 c\end{array}$ & \\
\hline Anabolic steroids & $\begin{array}{l}0.10(-0.02-0.23) ; \\
N^{1}=33 i \text { versus } 30 c\end{array}$ & $\begin{array}{l}4.00(-5.28-13.28) ; \\
N^{1}=33 i \text { versus } 30 \mathrm{c}\end{array}$ & & & & $\begin{array}{c}-63.0(-91.09--34.91) ; \\
N^{1}=10 i \text { versus } 7 c\end{array}$ & \\
\hline Growth hormone & $\begin{array}{l}0.05(0.01-0.09) ; \\
\mathrm{N}^{2}=18 \mathrm{i} \text { versus } 18 \mathrm{c}\end{array}$ & $\begin{array}{c}-11.00(-26.48-4.48) ; \\
N^{1}=8 \mathrm{i} \text { versus } 8 \mathrm{c}\end{array}$ & & & & $\begin{array}{c}-26.21(-148.92-96.50)^{\#} ; \\
N^{2}=22 i \text { versus } 23 c\end{array}$ & \\
\hline Vitamin D & $\begin{array}{c}0.64(0.07-1.22) ; \\
\mathrm{N}^{1}=25 i \text { versus } 24 \mathrm{c}\end{array}$ & $\begin{array}{l}7.00(-0.28-14.28) ; \\
N^{1}=25 i \text { versus } 24 c\end{array}$ & & & & $\begin{array}{l}29.0(-7.62-65.62) ; \\
\mathrm{N}^{1}=25 i \text { versus } 24 \mathrm{c}\end{array}$ & \\
\hline Hypertonic saline & & & & & & $\begin{array}{c}-190.0(-247.34--132.66) ; \\
N^{1}=30 i \text { versus } 27 c\end{array}$ & \\
\hline \multicolumn{8}{|l|}{ Nutritional supplementation } \\
\hline Proteins/fats & $\begin{array}{l}-0.03(-0.12-0.06) ; \\
\mathrm{N}^{1}=38 \text { i versus } 42 \mathrm{c}\end{array}$ & $\begin{array}{l}9.00(4.32-13.68) ; \\
N^{1}=38 i \text { versus } 42 c\end{array}$ & $\begin{array}{l}2.86(-2.00-7.72) \\
\mathrm{N}^{1}=10 \mathrm{i} \text { versus } 10 \mathrm{c}\end{array}$ & $\begin{array}{c}-17.40(-43.52-8.72) ; \\
N^{1}=25 i \text { versus } 35 \mathrm{c}\end{array}$ & & $\begin{array}{c}82.70(-38.59-203.99) \\
N^{1}=16 i \text { versus } 10 \mathrm{c}\end{array}$ & \\
\hline Creatine & $\begin{array}{l}0.14(-0.02-0.29) ; \\
N^{1}=14 i \text { versus } 11 \mathrm{c}\end{array}$ & $\begin{array}{l}4.29(-9.53-18.11) ; \\
N^{1}=14 i \text { versus } 11 \mathrm{c}\end{array}$ & & $\begin{array}{c}-3.62(-31.75-24.52) ; \\
N^{2}=52 i \text { versus } 53 c\end{array}$ & $\begin{array}{c}-0.67(-2.96-1.63) \mathrm{min} ; \\
\mathrm{N}^{2}=52 i \text { versus } 53 \mathrm{c}\end{array}$ & & \\
\hline \multicolumn{8}{|l|}{ Amino acids } \\
\hline \multicolumn{8}{|l|}{ Breathing exercises } \\
\hline Inspiratory muscle training & $\begin{array}{c}0.48(-0.17-1.14) \text { units"; } \\
N^{1}=20 \text { versus } 17 c\end{array}$ & & & & & $\begin{array}{l}29.68(-9.48-68.84) ; \\
N^{2}=34 \text { i versus } 30 \mathrm{c}\end{array}$ & \\
\hline Breathing retraining & & & & & & $\begin{array}{c}-12.58(-35.93-10.77) ; \\
N^{1}=20 i \text { versus } 20 \mathrm{c}\end{array}$ & \\
\hline Other & & & & $\begin{array}{l}3.20(-24.06-30.46) ; \\
N^{1}=16 i \text { versus } 25 \mathrm{c}\end{array}$ & & $\begin{array}{c}64.80(-12.94-142.54) ; \\
N^{1}=10 \mathrm{i} \text { versus } 10 \mathrm{c}\end{array}$ & \\
\hline
\end{tabular}

Data are presented as mean difference (95\% CI), where the mean difference is the change from baseline and a positive effect estimate favours intervention ("add-on" therapies). Data from individual studies may appear more than once across different outcomes (columns). $V^{\prime} \mathrm{O}_{2}$ peak: peak oxygen uptake; ISWT: incremental shuttle walk test; ESWT: endurance shuttle walk test; 6MWT: 6-min walk test; 12MWT: 12-min walk test; LL: lower limb; UL: upper limb; NIV: noninvasive ventilation; $\mathrm{N}^{\mathrm{x}}$ : number of participants (x is number of studies); i: intervention group; c: control group. ${ }^{\#}$ : random effects model; ${ }^{\text {I: }}$ standardised mean difference. Bold indicates statistically significant treatment effect. 
"additional exercise training" subcategories revealed that the upper limit of the confidence interval $(27.63 \mathrm{~m})$ was within the range of the MID for the 6MWT. Importantly, the study findings do not detract from the potential clinical benefits of "add-on" therapies on outcomes such as symptoms or quality of life. While improvement in such outcomes is unlikely to be mediated exclusively by extrapulmonary (e.g. musculoskeletal) physiological adaptations in response to exercise training, associated alterations in factors such as breathing pattern or subjective control of dyspnoea remain clinically important. Such benefits have been clearly documented in the pulmonary rehabilitation literature [2].

The review also provides evidence from individual studies of negative effects of hypertonic saline and anabolic steroids on 6MWT response, with data from two studies highlighting reductions greatly exceeding the MID [46, 53]. Importantly, supplementation of anabolic steroids is primarily used to enhance muscle strength [87]. While a significant decrease in muscle strength or function would not be an expected consequence of supplementation, it should also not be assumed that exercise capacity would be guaranteed to improve as a result of a pharmacological intervention targeting the cellular genesis of hypertrophic muscle responses. Unless new data emerge, demonstrating opposing findings, the continued use of these interventions in conjunction with standard exercise training in pulmonary rehabilitation appears inadvisable.

The general lack of observed benefit across the breadth of interventions and outcomes could have been affected by three factors. First, as pulmonary rehabilitation comprising exercise training is already a highly effective treatment for patients with COPD [1], further improvements in exercise capacity via "add-on" modalities may be challenging to obtain. This is particularly relevant when one considers that pulmonary rehabilitation targets extrapulmonary features of COPD, particularly muscle composition and function, which may have realistic ceilings in terms of the magnitude of expected improvement within a short time-frame. This raises subsequent issues regarding attainable statistical power and sample size estimations for future clinical trials of "add-on" therapies to exercise training in COPD. In such studies, conventional MID thresholds of improvement may not be the most appropriate measure of clinical importance if the underlying exercise training co-intervention is expected to be highly effective for the same outcome. Smaller subsequent levels of improvement (e.g. $+15 \mathrm{~m}$ on top of $+30 \mathrm{~m}$ 6MWT change) could, for example, be clinically meaningful. Identifying the importance of such additional effects for clinicians and healthcare providers could perhaps be facilitated via use of more intuitive research metrics than those commonly seen in the relevant literature. For example, data regarding the "number needed to treat" (the number of patients that need to be treated to have a meaningful impact on one person) were not available in any study included in the review, yet this offers a simple estimate of clinical value in consideration of broader issues such as cost-effectiveness.

Secondly, although the effects of "add-on" therapies on exercise capacity were reported for all interventions included in the review, not all interventions primarily target direct improvements in exercise capacity (e.g. psychotherapy or self-management). Interventions such as NIV, for example, aim to ameliorate negative physiological responses to exercise training (such as ventilation limitation) and reduce symptom burden. These indirect influences on exercise training responses may not be fully captured within measures of exercise performance alone. Related to this, the fidelity of interventions must be carefully considered, to ensure they are applied appropriately (i.e. to achieve their stated purpose) and deemed adequately acceptable to patients (i.e. to ensure adherence). This may have limited the effectiveness of some interventions in this review, such as NIV applied at very low intensities during training. It would be interesting to speculate whether improvement of symptoms during exercise would improve treatment adherence; however, this was not explicitly examined in the present review. These issues highlight the importance of carefully selected outcome reporting in future investigations, and the need for consensus among researchers, clinicians and policy makers to identify the outcomes considered most important to inform clinical practice.

Thirdly, pulmonary rehabilitation studies rarely account for significant disease heterogeneity in COPD. The "one size fits all" model of care applied in most clinical trials seems to derive favourable, clinically important benefits to COPD patients on average; however, further improvements with "add-on" therapies may only be possible in specific patient subgroups. Intuition might suggest the use of highly specific eligibility criteria well suited to "add-on" interventions might be associated with positive results. This was not, however, supported by the findings of our secondary analysis. Interpretation of these exemplar studies was limited by small sample size and low number of studies adopting this approach. This, in itself, is a highly important outcome of the present review that should inform future research. While such patient-centred studies may not yield positive results on exercise capacity, they are likely to confer more accurate information about the true clinical utility of interventions, as they represent the best available estimate of effectiveness in well-defined patient subgroups most likely to derive benefit. Further studies should also strive to achieve adequate statistical power for key outcomes, as small sample sizes and resultant under-powering was a likely contributor to the lack of significant findings observed in this review. 
Studies in specific COPD clinical phenotypes are more difficult to conduct, as recruitment is typically slower, with a potential impact upon statistical power. Such studies may confer less external validity than broader studies, but the potential for clinically applicable findings is increased. The development of strong multicentre, international collaborations appears essential in order to conduct high-quality large randomised controlled trials to advance future clinical practice. The diverse organisation of pulmonary rehabilitation programmes across the world [88] can impede such studies; however, standardisation of pulmonary rehabilitation research outcomes, similar to the widespread use of the forced expiratory volume in pharmacological studies, could help overcome this issue. Adoption of "mandatory" outcomes for reporting exercise performance could aid this process, such as the extensive use and importance of the $6 \mathrm{MWT}$ documented in recent pulmonary rehabilitation guidelines [85, 89].

\section{Limitations}

While pulmonary rehabilitation benefits are well documented on outcomes such as exercise capacity, quality of life and healthcare utilisation $[2,3]$, the scope of the present review was restricted to outcomes of exercise capacity, admittedly an essential outcome in pulmonary rehabilitation. This was necessary due to the large number of anticipated studies included in the review. The findings therefore relate exclusively to this essential mechanism underpinning exercise training benefits. Potential effects of "add-on" therapies on other important outcomes, such as symptoms or quality of life, should not be extrapolated from our data.

We also included end-point data where changes from baseline data were unavailable. This significantly increased the volume of data and opportunity for meta-analysis to improve findings from the earlier review in this area [7]. A known negative effect of this method, however, is relative overweighting to data from studies contributing change data compared with those contributing end-point data. This is due to the typically narrower confidence intervals around change data compared with end-point data. The consistency of results across clinical trials (figure 3), however, suggests this did not adversely affect the review findings.

The heterogeneous array of interventions and limited opportunity for meta-analysis for some outcomes meant that interpretation of some findings and identification of clinical implications was difficult. Some interventions within the same group have similar goals but work through different mechanisms (e.g. neuromuscular electrical stimulation, vibration training and eccentric training to improve muscle function within the "additional training modalities" group). Significant statistical heterogeneity was observed within some outcomes (tables 3 and 4). Despite attempts to maximise opportunities for data pooling, the resultant number of included participants in the meta-analyses was quite low. This was particularly evident in the analysis of carefully selected COPD subgroups (i.e. patient-centred studies) and reinforced the difficulty of drawing clinically meaningful conclusions from the large body of literature in this field. Given the observed rate of attrition across studies (table 1), it also seems important that future studies report findings with strict adherence to the principle of intention-to-treat, in order to clarify the true value of treatments applied in the context of "real life" settings. These limitations offer strong support for change in the approach taken towards future investigations in this area. Exercise training represents a complex intervention and standardisation of programme length, intensity and basic outcome reporting would greatly assist the interpretation of future analyses.

\section{Conclusion}

Results from our large data synthesis of a diverse array of "add-on" physical, pharmacological, nutritional and behavioural interventions demonstrate minimal clinically important improvements in exercise capacity above those expected from conventional exercise training in pulmonary rehabilitation. This provides a compelling argument discouraging continued attempts to investigate the effectiveness of different "add-on" modalities on exercise capacity using methodologies similar to those included in this review. This does not, however, mean it is unimportant to conduct further studies of "add-on" therapies in this patient group, particularly evaluation of their effect on other outcomes from those covered in this review. Identification of the true value of such therapies may, however, not emerge until studies are carefully designed and implemented in highly selective patient groups using the most appropriate outcomes.

As many pulmonary rehabilitation benefits are driven by physiological change in the peripheral skeletal muscles in response to exercise training, it seems sensible for future clinical trials of interventions in this area to be based upon a strong physiological foundation for specific muscular targets (whether at cellular tissue sites or a physical function level) in select clinical COPD phenotypes. Such interventions should be associated with markers of improvement in relevant outcomes such as exercise capacity and muscle force. Definitive answers regarding the future of pulmonary rehabilitation are therefore only likely to emerge from carefully designed investigations of specific interventions in select patients in larger multicentre studies. 


\section{Acknowledgements}

The authors would like to thank François Maltais, Louis Laviolette (both Centre de Recherche, Institut Universitaire de Cardiologie et de Pneumologie de Quebec, Quebec, QC, Canada) and Mauricio Jamami (Departamento de Fisioterapia, Universidade Federal de São Carlos, São Carlos, Brazil) for providing data upon request for this systematic review. We also would like to thank Peter Spiegler (Pulmonary and Critical Care Medicine, Winthrop University Hospital, Mineola, NY, USA) for providing us with information regarding their exercise training programme.

\section{References}

1 Spruit MA, Singh SJ, Garvey C, et al. An official American Thoracic Society/European Respiratory Society statement: key concepts and advances in pulmonary rehabilitation. Am J Respir Crit Care Med 2013; 188: e13-e64.

2 McCarthy B, Casey D, Devane D, et al. Pulmonary rehabilitation for chronic obstructive pulmonary disease. Cochrane Database Syst Rev 2015; 2: CD003793.

3 Puhan MA, Gimeno-Santos E, Scharplatz M, et al. Pulmonary rehabilitation following exacerbations of chronic obstructive pulmonary disease. Cochrane Database Syst Rev 2011; 10: CD005305.

4 Casaburi R, Porszasz J, Burns MR, et al. Physiologic benefits of exercise training in rehabilitation of patients with severe chronic obstructive pulmonary disease. Am J Respir Crit Care Med 1997; 155: 1541-1551.

5 Whittom F, Jobin J, Simard PM, et al. Histochemical and morphological characteristics of the vastus lateralis muscle in patients with chronic obstructive pulmonary disease. Med Sci Sports Exerc 1998; 30: 1467-1474.

6 Maltais F, LeBlanc P, Simard C, et al. Skeletal muscle adaptation to endurance training in patients with chronic obstructive pulmonary disease. Am J Respir Crit Care Med 1996; 154: 442-447.

7 Puhan MA, Schünemann HJ, Frey M, et al. Value of supplemental interventions to enhance the effectiveness of physical exercise during respiratory rehabilitation in COPD patients. A systematic review. Respir Res 2004; 5: 25.

8 de Morton NA. The PEDro scale is a valid measure of the methodological quality of clinical trials: a demographic study. Aust J Physiother 2009; 55: 129-133.

9 Benton MJ, Wagner CL. Effect of single-set resistance training on quality of life in COPD patients enrolled in pulmonary rehabilitation. Respir Care 2013; 58: 487-493.

10 Vonbank K, Strasser B, Mondrzyk J, et al. Strength training increases maximum working capacity in patients with COPD - randomized clinical trial comparing three training modalities. Respir Med 2012; 106: 557-563.

11 Bernard S, Whittom F, LeBlanc P, et al. Aerobic and strength training in patients with chronic obstructive pulmonary disease. Am J Respir Crit Care Med 1999; 159: 896-901.

12 Dourado VZ, Tanni SE, Antunes LC, et al. Effect of three exercise programs on patients with chronic obstructive pulmonary disease. Braz J Med Biol Res 2009; 42: 263-271

13 Phillips WT, Benton MJ, Wagner CL, et al. The effect of single set resistance training on strength and functional fitness in pulmonary rehabilitation patients. J Cardiopulm Rehabil 2006; 26: 330-337.

14 Mador MJ, Bozkanat E, Aggarwal A, et al. Endurance and strength training in patients with COPD. Chest 2004; 125: 2036-2045.

15 Würtemberger G, Bastian K. Funktionelle Effekte unterschiedlicher Trainingsformen bei Patienten mit COPD [Functional effects of different training in patients with COPD]. Pneumologie 2001; 55: 553-562.

16 Alexander JL, Phillips WT, Wagner CL. The effect of strength training on functional fitness in older patients with chronic lung disease enrolled in pulmonary rehabilitation. Rehabil Nurs 2008; 33: 91-97.

17 Holland AE, Hill CJ, Nehez E, et al. Does unsupported upper limb exercise training improve symptoms and quality of life for patients with chronic obstructive pulmonary disease? J Cardiopulm Rehabil 2004; 24: 422-427.

18 Subin, Rao V, Prem V, et al. Effect of upper limb, lower limb and combined training on health-related quality of life in COPD. Lung India 2010; 27: 4-7.

19 Sívori M, Rhodius E, Kaplan P, et al. Entrenamiento muscular en la enfermedad pulmonar obstructiva cronica severa. Estudio comparativo del entrenamiento aerobico de miembros inferiores vs. combinacion con miembros superiors. [Exercise training in chronic obstructive pulmonary disease. Comparative study of aerobic training of lower limbs vs. combination with upper limbs.] Medicina 1998; 58: 717-727.

20 Costi S, Crisafulli E, Antoni FD, et al. Effects of unsupported upper extremity exercise training in patients with COPD: a randomized clinical trial. Chest 2009; 136: 387-395.

21 Vivodtzev I, Pépin JL, Vottero G, et al. Improvement in quadriceps strength and dyspnea in daily tasks after 1 month of electrical stimulation in severely deconditioned and malnourished COPD. Chest 2006; 129: 1540-1548.

22 Rooyackers JM, Berkeljon DA, Folgering HT. Eccentric exercise training in patients with chronic obstructive pulmonary disease. Int J Rehabil Res 2003; 26: 47-49.

23 Gloeckl R, Heinzelmann I, Baeuerle S, et al. Effects of whole body vibration in patients with chronic obstructive pulmonary disease - a randomized controlled trial. Respir Med 2012; 106: 75-83.

24 Johnson JE, Gavin DJ, Adams-Dramiga S. Effects of training with heliox and noninvasive positive pressure ventilation on exercise ability in patients with severe COPD. Chest 2002; 122: 464-472.

25 Toledo A, Borghi-Silva A, Sampaio LM, et al. The impact of noninvasive ventilation during the physical training in patients with moderate-to-severe chronic obstructive pulmonary disease (COPD). Clinics 2007; 62: 113-120.

26 Reuveny R, Ben-Dov I, Gaides M, et al. Ventilatory support during training improves training benefit in severe chronic airway obstruction. Isr Med Assoc J 2005; 7: 151-155.

27 Costes F, Agresti A, Court-Fortune I, et al. Noninvasive ventilation during exercise training improves exercise tolerance in patients with chronic obstructive pulmonary disease. J Cardiopulm Rehabil 2003; 23: 307-313.

28 van 't Hul A, Gosselink R, Hollander P, et al. Training with inspiratory pressure support in patients with severe COPD. Eur Respir J 2006; 27: 65-72.

29 Hawkins P, Johnson LC, Nikoletou D, et al. Proportional assist ventilation as an aid to exercise training in severe chronic obstructive pulmonary disease. Thorax 2002; 57: 853-859.

30 Bianchi L, Foglio K, Porta R, et al. Lack of additional effect of adjunct of assisted ventilation to pulmonary rehabilitation in mild COPD patients. Respir Med 2002; 96: 359-367.

31 Garrod R, Mikelsons C, Paul EA, et al. Randomized controlled trial of domiciliary noninvasive positive pressure ventilation and physical training in severe chronic obstructive pulmonary disease. Am J Respir Crit Care Med 2000; 162: 1335-1341 
32 Duiverman ML, Wempe JB, Bladder G, et al. Nocturnal non-invasive ventilation in addition to rehabilitation in hypercapnic patients with COPD. Thorax 2008; 63: 1052-1057.

33 Scorsone D, Bartolini S, Saporiti R, et al. Does a low-density gas mixture or oxygen supplementation improve exercise training in COPD? Chest 2010; 138: 1133-1139.

34 Emtner M, Porszasz J, Burns M, et al. Benefits of supplemental oxygen in exercise training in nonhypoxemic chronic obstructive pulmonary disease patients. Am J Respir Crit Care Med 2003; 168: 1034-1042.

35 Garrod R, Paul EA, Wedzicha JA. Supplemental oxygen during pulmonary rehabilitation in patients with COPD with exercise hypoxaemia. Thorax 2000; 55: 539-543.

36 Wadell K, Henriksson-Larsén K, Lundgren R. Physical training with and without oxygen in patients with chronic obstructive pulmonary disease and exercise-induced hypoxaemia. J Rehabil Med 2001; 33: 200-205.

37 Dyer F, Callaghan J, Cheema K, et al. Ambulatory oxygen improves the effectiveness of pulmonary rehabilitation in selected patients with chronic obstructive pulmonary disease. Chron Respir Dis 2012; 9: 83-91.

38 Rooyackers JM, Dekhuijzen PN, van Herwaarden CL, et al. Training with supplemental oxygen in patients with COPD and hypoxaemia at peak exercise. Eur Respir J 1997; 10: 1278-1284.

39 Bjørgen S, Helgerud J, Husby V, et al. Aerobic high intensity one-legged interval cycling improves peak oxygen uptake in chronic obstructive pulmonary disease patients; no additional effect from hyperoxia. Int J Sports Med 2009; 30: 872-878.

40 Ringbaek T, Martinez G, Lange P. The long-term effect of ambulatory oxygen in normoxaemic COPD patients: a randomised study. Chron Respir Dis 2013; 10: 77-84.

41 Eves ND, Sandmeyer LC, Wong EY, et al. Helium-hyperoxia: a novel intervention to improve the benefits of pulmonary rehabilitation for patients with COPD. Chest 2009; 135: 609-618.

42 Pasqua F, Biscione G, Crigna G, et al. Combining triple therapy and pulmonary rehabilitation in patients with advanced COPD: a pilot study. Respir Med 2010; 104: 412-417.

43 Ambrosino N, Foglio K, Balzano G, et al. Tiotropium and exercise training in COPD patients: effects on dyspnea and exercise tolerance. Int J Chron Obstruct Pulmon Dis 2008; 3: 771-780.

44 Casaburi R, Kukafka D, Cooper CB, et al. Improvement in exercise tolerance with the combination of tiotropium and pulmonary rehabilitation in patients with COPD. Chest 2005; 127: 809-817.

45 Creutzberg EC, Wouters EF, Mostert R, et al. A role for anabolic steroids in the rehabilitation of patients with COPD? A double-blind, placebo-controlled, randomized trial. Chest 2003; 124: 1733-1742.

46 Ferreira IM, Verreschi IT, Nery LE, et al. The influence of 6 months of oral anabolic steroids on body mass and respiratory muscles in undernourished COPD patients. Chest 1998; 114: 19-28.

47 Miki K, Maekura R, Nagaya N, et al. Ghrelin treatment of cachectic patients with chronic obstructive pulmonary disease: a multicenter, randomized, double-blind, placebo-controlled trial. PLoS One 2012; 7: e35708.

48 Burdet L, de Muralt B, Schutz Y, et al. Administration of growth hormone to underweight patients with chronic obstructive pulmonary disease. A prospective, randomized, controlled study. Am J Respir Crit Care Med 1997; 156: 1800-1806.

49 Miki K, Maekura R, Nagaya N, et al. Effects of ghrelin treatment on exercise capacity in underweight COPD patients: a substudy of a multicenter, randomized, double-blind, placebo-controlled trial of ghrelin treatment. BMC Pulm Med 2013; 13: 37.

50 Hornikx M, Van Remoortel H, Lehouck A, et al. Vitamin D supplementation during rehabilitation in COPD: a secondary analysis of a randomized trial. Respir Res 2012; 13: 84

51 Blanco I, Santos S, Gea J, et al. Sildenafil to improve respiratory rehabilitation outcomes in COPD: a controlled trial. Eur Respir J 2013; 42: 982-992.

52 Satta A, Grandi M, Landoni CV, et al. Effects of ubidecarenone in an exercise training program for patients with chronic obstructive pulmonary diseases. Clin Ther 1991; 13: 754-757.

53 Valderramas SR, Atallah AN. Effectiveness and safety of hypertonic saline inhalation combined with exercise training in patients with chronic obstructive pulmonary disease: a randomized trial. Respir Care 2009; 54: 327-333.

54 Laviolette L, Lands LC, Dauletbaev N, et al. Combined effect of dietary supplementation with pressurized whey and exercise training in chronic obstructive pulmonary disease: a randomized, controlled, double-blind pilot study. J Med Food 2010; 13: 589-598.

55 Sugawara K, Takahashi H, Kashiwagura T, et al. Effect of anti-inflammatory supplementation with whey peptide and exercise therapy in patients with COPD. Respir Med 2012; 106: 1526-1534.

56 Steiner MC, Barton RL, Singh SJ, et al. Nutritional enhancement of exercise performance in chronic obstructive pulmonary disease: a randomised controlled trial. Thorax 2003; 58: 745-751.

57 Gurgun A, Deniz S, Argin M, et al. Effects of nutritional supplementation combined with conventional pulmonary rehabilitation in muscle-wasted chronic obstructive pulmonary disease: a prospective, randomized and controlled study. Respirology 2013; 18: 495-500.

58 Broekhuizen R, Wouters EF, Creutzberg EC, et al. Polyunsaturated fatty acids improve exercise capacity in chronic obstructive pulmonary disease. Thorax 2005; 60: 376-382.

59 Deacon SJ, Vincent EE, Greenhaff PL, et al. Randomized controlled trial of dietary creatine as an adjunct therapy to physical training in chronic obstructive pulmonary disease. Am J Respir Crit Care Med 2008; 178: $233-239$.

60 Faager G, Söderlund K, Sköld CM, et al. Creatine supplementation and physical training in patients with COPD: a double blind, placebo-controlled study. Int J Chron Obstruct Pulmon Dis 2006; 1: 445-453.

61 Fuld JP, Kilduff LP, Neder JA, et al. Creatine supplementation during pulmonary rehabilitation in chronic obstructive pulmonary disease. Thorax 2005; 60: 531-537.

62 Menier R, Talmud J, Laplaud D, et al. Branched-chain aminoacids and retraining of patients with chronic obstructive lung disease. J Sports Med Phys Fitness 2001; 41: 500-504.

63 Borghi-Silva A, Baldissera V, Sampaio LM, et al. L-carnitine as an ergogenic aid for patients with chronic obstructive pulmonary disease submitted to whole-body and respiratory muscle training programs. Braz J Med Biol Res 2006; 39: 465-474.

64 Magadle R, McConnell AK, Beckerman M, et al. Inspiratory muscle training in pulmonary rehabilitation program in COPD patients. Respir Med 2007; 101: 1500-1505.

65 Mador MJ, Deniz O, Aggarwal A, et al. Effect of respiratory muscle endurance training in patients with COPD undergoing pulmonary rehabilitation. Chest 2005; 128: 1216-1224. 
Larson JL, Covey MK, Wirtz SE, et al. Cycle ergometer and inspiratory muscle training in chronic obstructive pulmonary disease. Am J Respir Crit Care Med 1999; 160: 500-507.

Berry MJ, Adair NE, Sevensky KS, et al. Inspiratory muscle training and whole-body reconditioning in chronic obstructive pulmonary disease. Am J Respir Crit Care Med 1996; 153: 1812-1816.

Wanke T, Formanek D, Lahrmann $\mathrm{H}$, et al. Effects of combined inspiratory muscle and cycle ergometer training on exercise performance in patients with COPD. Eur Respir J 1994; 7: 2205-2211.

Weiner P, Azgad Y, Ganam R. Inspiratory muscle training combined with general exercise reconditioning in patients with COPD. Chest 1992; 102: 1351-1356.

Dekhuijzen PN, Folgering HT, van Herwaarden CL. Target-flow inspiratory muscle training during pulmonary rehabilitation in patients with COPD. Chest 1991; 99: 128-133.

1 Kunikoshita LN, Silva YP, Silva TLP, et al. Efeitos de três programas de fisioterapia respiratória (PFR) em portadores de DPOC [Effects of three respiratory physical therapy programs on patients with COPD]. Braz J Phys Ther 2006; 10: 449-455.

2 Sykes K, Hang HW. Inspiratory muscle training in the treatment of chronic obstructive pulmonary disease: randomized controlled trial. Am J Recreat Ther 2005; 4: 39-48.

3 Goldstein R, De Rosie J, Long S, et al. Applicability of a threshold loading device for inspiratory muscle testing and training in patients with COPD. Chest 1989; 96: 564-571.

Collins EG, Langbein WE, Fehr L, et al. Can ventilation-feedback training augment exercise tolerance in patients with chronic obstructive pulmonary disease? Am J Respir Crit Care Med 2008; 177: 844-852.

van Gestel AJ, Kohler M, Steier J, et al. The effects of controlled breathing during pulmonary rehabilitation in patients with COPD. Respiration 2012; 83: 115-124.

Carrieri-Kohlman V, Gormley JM, Douglas MK, et al. Exercise training decreases dyspnea and the distress and anxiety associated with it. Monitoring alone may be as effective as coaching. Chest 1996; 110: 1526-1535.

7 Zanotti E, Berardinelli P, Bizzarri C, et al. Osteopathic manipulative treatment effectiveness in severe chronic obstructive pulmonary disease: a pilot study. Complement Ther Med 2012; 20: 16-22.

8 Alexander JL, Wagner CL. Is harmonica playing an effective adjunct therapy to pulmonary rehabilitation? Rehabil Nurs 2012; 37: 207-212.

9 de Godoy DV, de Godoy RF. A randomized controlled trial of the effect of psychotherapy on anxiety and depression in chronic obstructive pulmonary disease. Arch Phys Med Rehabil 2003; 84: 1154-1157.

0 Sharifabad MA, Hurewitz A, Spiegler P, et al. Written disclosure therapy for patients with chronic lung disease undergoing pulmonary rehabilitation. J Cardiopulm Rehabil Prev 2010; 30: 340-345.

1 Norweg AM, Whiteson J, Malgady R, et al. The effectiveness of different combinations of pulmonary rehabilitation program components: a randomized controlled trial. Chest 2005; 128: 663-672.

Deering BM, Fullen B, Egan C, et al. Acupuncture as an adjunct to pulmonary rehabilitation. $J$ Cardiopulm Rehabil Prev 2011; 31: 392-399.

3 Higgins JPT, Green S, eds. Cochrane Handbook for Systematic Reviews of Interventions. Version 5.1.0 (updated March 2011). London, The Cochrane Collaboration, 2011. Available from: www.cochrane-handbook.org

4 Bolton CE, Bevan-Smith EF, Blakey JD, et al. British Thoracic Society guideline on pulmonary rehabilitation in adults. Thorax 2013; 68: Suppl. 2, ii1-ii30.

Holland AE, Spruit MA, Troosters T, et al. An official European Respiratory Society/American Thoracic Society technical standard: field walking tests in chronic respiratory disease. Eur Respir J 2014; 44: 1428-1446.

86 Singh SJ, Puhan MA, Andrianopoulos V, et al. An official systematic review of the European Respiratory Society/ American Thoracic Society: measurement properties of field walking tests in chronic respiratory disease. Eur Respir J 2014; 44: 1447-1478.

7 Casaburi R. Rationale for anabolic therapy to facilitate rehabilitation in chronic obstructive pulmonary disease. Baillieres Clin Endocrinol Metab 1998; 12: 407-418.

8 Spruit MA, Pitta F, Garvey C, et al. Differences in content and organisational aspects of pulmonary rehabilitation programmes. Eur Respir J 2014; 43: 1326-1337.

Watz H, Pitta F, Rochester CL, et al. An official European Respiratory Society statement on physical activity in COPD. Eur Respir J 2014; 44: 1521-1537. 